\title{
AC Followed by Pertuzumab/Trastuzumab/Paclitaxel Regimen
}

National Cancer Institute

\section{Source}

National Cancer Institute. AC Followed by Pertuzumab/Trastuzumab/Paclitaxel Regimen NCI Thesaurus. Code C138032.

A chemotherapy regimen consisting of cyclophosphamide and doxorubicin followed by pertuzumab, trastuzumab, and paclitaxel, used as a neoadjuvant or adjuvant treatment for HER-2/neu-positive breast cancer. 